\title{
Detection of Non-Symmetrical Damage in Smart Plate-Like Structures ${ }^{1}$
}

\author{
H.T. Banks and P.R. Emeric \\ Center for Research in Scientific Computation \\ North Carolina State University \\ Box 8205 \\ Raleigh, NC 27695-8205
}

January 30, 1998

\begin{abstract}
A two-dimensional model for in-plane vibrations of a cantilever plate with a non-symmetrical damage is used in the context of defect identification in materials with piezoelectric ceramic patches bonded to their surface. These patches can act both as actuators and sensors in a self-analyzing fashion, which is a characteristic of smart materials. A Galerkin method is used to approximate the dynamic response of these structures. The natural frequency shifts due to the damage are estimated numerically and compared to experimental data obtained from tests on cantilever aluminum plate-like structures damaged at different locations with defects of different depths. The damage location and extent are determined by an enhanced least square identification method. Efficacy of the frequency shift based algorithms is demonstrated using experimental data.
\end{abstract}

\footnotetext{
${ }^{1}$ This research was funded in part by the U.S. Air Force Office of Scientific Research under grant number AFOSR F49620-95-1-0236. Parts of the research reported here were conducted while the authors were visiting scientists at the Institute for Computer Applications in Science and Engineering (ICASE) which is granted under NASA contracts NAS1-97046 and NAS1-19480.
} 


\section{Introduction}

The focus of this report is the detection and characterization of non-symmetrical damage in smart cantilever plate-like structures. Smart materials are used in a growing number of commercial applications such as the aerospace, automotive and machine-tool industries. In particular, smart structures with self actuating and sensing capabilities have potential applications in non-destructive evaluation of damage $[2,14]$. Such capabilities can be achieved by bonding piezoelectric ceramic patches to the surface of a structure to be characterized. When an electric field is applied, piezoceramic patches induce strains in the material to which they are bonded and, conversely, they produce a voltage when a deformation occurs in the material $[3,4,5]$. As a consequence, these patches can act both as actuators and sensors, providing the host structure with smart material capabilities.

The premise of the detection method is that damage to a structure will induce changes in the natural frequencies which can be obtained by measurement of the vibration response. A damage corresponds to a local loss of stiffness, which lowers the natural frequencies of the structure. Modal methods are typically easy to implement and are already widely used in civil engineering applications $[6,7,8]$. In the context of damage detection in beam-like structures, the damaged beams are often modeled by a 1-dimensional (1-D) equation with a massless rotational spring introduced at the location of the damage $[9,10,11,12,13]$. The damage identification is achieved by comparison of simulated natural frequencies of the damaged structure to the measured ones obtained from dynamic testing.

Damage detection methods based on 1-D models are popular but have known shortcomings. For instance, the position of the defects with respect to the maximum curvature of the mode shapes is a determining factor. Krawczuk and Ostachowicz [11] found that the influence of a crack located at a point of minimum curvature with a dimension ratio of less than 0.3 of the total height had practically no effect on the first three natural frequencies. Banks et al [14] have shown in detail the lack of sensitivity for 1-D systems to mass and frequency changes. As an alternate method to modal analysis for the characterization of damage, they proposed a direct time domain estimation method of spatially varying physical parameters of the structures. However, it should be noted that, for civil engineering applications, the lack of sensitivity of modal methods to small defects is not a severe limitation since most structures are redundant and small defects are not critical.

Another important limitation when using 1-D models is that the shape of the defect cannot be recovered [22]. The case of cracks is particularly challenging since the compliance introduced at the location of the damage really represents two symmetrically located cracks. Christides and Barr [16] developed a theory with a symmetrically cracked Euler-Bernoulli beam. They assumed that the cracks remained open at all times. Fu and Mimovich [15] presented an experimentally validated modal frequency method to diagnose fracture in beams. They successfully located damage to within $3 \%$ of the length but report significant errors in the determination of the crack intensity. They attributed the errors to the differences in the effects of a theoretical crack and that of a slit. Cawley and Ray [17] studied the importance of defect width on the natural frequencies of a structure. They concluded, like Fu and Mimovich, that cracks and

slits cannot be investigated in the same manner. Brandon and Abraham [18, 19] proposed a method to predict the vibration properties of a beam with a breathing transverse crack by using a bilinear model for the open and closed cases. Gudmundson [20] solved the problem 
of the crack closure by statically preloading the specimens. Qian et al [21] developed their model by assuming that the structure was undamaged when the crack was closed. In their investigation, they only considered the first three natural frequencies and pointed out that, in most cases, higher order modal information was needed to determine the crack location. In a numerical study of structural damage assessment from natural frequency measurement, Hassiotis and Jeong [22] used the first five natural frequencies for a cantilever beam and the first ten for a frame. Their method proved successful in identifying both location and severity by using exact or noise-polluted simulated natural frequency data. This suggests that lower frequencies are suited for damage detection and that higher ones can improve the identification.

In the present work, we are only interested in the characterization of non-symmetrical defects with finite width. To overcome the shortcomings of methods based on Euler-Bernoulli and Timoshenko theories, we propose a 2-D model [1] to predict the vibration response of beam-like structures. For the identification of damage, we propose to use both lower and higher order modal information. Such information can be easily obtained with a dynamic testing procedure taking advantage of the self actuating and sensing capabilities of piezo-electric patches. In the first part of this work, a 2-D model for the in-plane vibrations of a damped linear elastic hookean cantilever plate with a non-symmetrical damage is summarized. Unfortunately, for most geometries and boundary conditions, including the case of interest, explicit solutions for the motion are not readily available. Approximation methods must be used. In the second part of this report, the model is discretized by using a Galerkin method with linear splines. The resulting finite dimensional matrix system is detailed. Then, in the third part, an experimental setup to measure the dynamic response of aluminum cantilever structures damaged at different locations with defects of different depths is presented. Finally, in the fourth section, the damage identification problem is formulated as a least square optimization problem. Numerical simulations and comparison with experimental data are discussed.

\section{$1 \quad$ Model}

\subsection{Physical Model}

Fig. 1 depicts a cross section $\Omega$ of the clamped plate-like structure with a non-symmetrical damage. $\Gamma$ is the boundary with no displacement. Motion is assumed to be longitudinal (in the $x$ direction) and transverse (in the $y$ direction). A groove in the $z$ direction represents the defect. Piezoelectric patches, denoted $\Omega_{p e}$, are symmetrically bonded to the upper and lower surfaces. The region $\Omega_{\mathcal{B}}=\Omega \cup \Omega_{p e}$ is the complete structure. The motion of the structure in the $(x, y)$ plane cannot be modeled accurately by classical thin beam or thin plate models. Such models assume a shell coordinate system with an unperturbed middle surface as the reference surface. The presence of non-symmetrical damage introduces a coupling of the motions in the $(x, y)$ plane which is not taken into account by these models. We model this coupled motion assuming a state of plane strain in the structure; that is, away from the edges, no displacement takes place in the $z$ direction. 


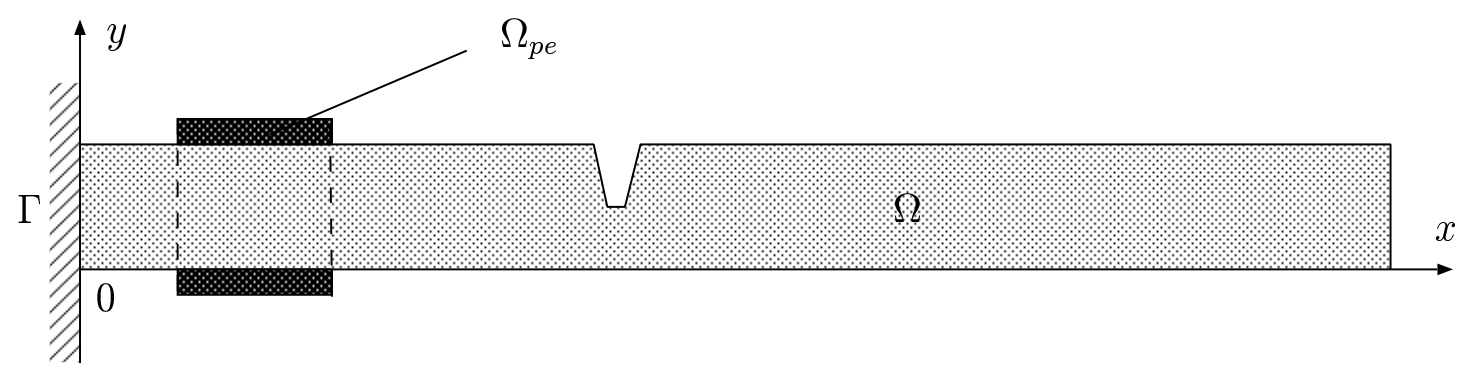

Figure 1: Cross section of damaged cantilever structure with piezoceramic patches

\subsection{Mathematical Model}

\subsubsection{Equations of Motion}

We combine force balancing with constitutive hypotheses to obtain the equations of motion for the displacements $U$ and $V$ in the $x$ and $y$ directions, respectively [1]. We obtain

$$
\left\{\begin{aligned}
\hat{\rho} \ddot{U}= & \frac{\hat{E}}{1+\hat{\nu}}\left(\frac{1-\hat{\nu}}{1-2 \hat{\nu}} U_{, x x}+\frac{1}{2} U_{, y y}+\frac{1}{2(1-2 \hat{\nu})} V_{, x y}\right) \\
& +\frac{\hat{C}_{D}}{1+\hat{\nu}}\left(\frac{1-\hat{\nu}}{1-2 \hat{\nu}} \dot{U}_{, x x}+\frac{1}{2} \dot{U}_{, y y}+\frac{1}{2(1-2 \hat{\nu})} \dot{V}_{, x y}\right)+\frac{q_{x}}{b} \\
\hat{\rho} \ddot{V}= & \frac{\hat{E}}{1+\hat{\nu}}\left(\frac{1-\hat{\nu}}{1-2 \hat{\nu}} V_{, y y}+\frac{1}{2} V_{, x x}+\frac{1}{2(1-2 \hat{\nu})} U_{, x y}\right) \\
& +\frac{\hat{C}_{D}}{1+\hat{\nu}}\left(\frac{1-\hat{\nu}}{1-2 \hat{\nu}} \dot{V}_{, y y}+\frac{1}{2} \dot{V}_{, x x}+\frac{1}{2(1-2 \hat{\nu})} \dot{U}_{, x y}\right)+\frac{q_{y}}{b}
\end{aligned}\right.
$$

where $b$ is the width in the $z$ direction, $q_{x}$ and $q_{y}$ are the horizontal and vertical components ${ }^{2}$ of an external force/unit area acting on the structure and $\dot{U}, \dot{V}$ and $\ddot{U}, \ddot{V}$ are the first and second time derivatives of the displacements $U$ and $V$. Here, $\hat{\rho}>0$ is the mass per unit volume, $\hat{C}_{D}>0$ is a damping coefficient, $\hat{E}>0$ is Young's modulus and $0 \leq \hat{\nu}<1 / 2$ is Poisson's ratio. These are locally constant material properties with definitions:

$$
\begin{array}{ll}
\hat{\rho}=\rho \chi_{\Omega}+\rho_{p e} \chi_{\Omega_{p e}}, & \hat{\nu}=\nu \chi_{\Omega}+\nu_{p e} \chi_{\Omega_{p e}} \\
\hat{C}_{D}=C_{D} \chi_{\Omega}+C_{D p e} \chi_{\Omega_{p e}}, & \hat{E}=E \chi_{\Omega}+E_{p e} \chi_{\Omega_{p e}}
\end{array}
$$

where the subscript ${ }_{p e}$ indicates the value for the piezoelectric patches. The functions $\chi_{\Omega}$ and $\chi_{\Omega_{p e}}$ are characteristic functions with definition

$$
\chi_{\Omega}=\left\{\begin{array}{ll}
1 & \text { in } \Omega \\
0 & \text { otherwise }
\end{array} \quad \text { and } \quad \chi_{\Omega_{p e}}= \begin{cases}1 & \text { in } \Omega_{p e} \\
0 & \text { otherwise }\end{cases}\right.
$$

\footnotetext{
${ }^{2}$ Throughout, subscripts such as those above do not represent derivatives; for partial derivatives, we shall use either $\frac{\partial U}{\partial x}$ or $U_{, x}$ whereas $q_{x}$ will denote the $x$ component of the force $q$.
} 


\subsubsection{External Forces}

The forces induced in the structure when a voltage is applied to the piezoelectric patches generally lack regularity (i.e. they contain terms involving $\delta$ and $\delta^{\prime}$ where $\delta$ is the Dirac delta function). The corresponding model and a mathematical formulation of the problem as a second order equation in time, detailed in [1], allow the discussion of existence and uniqueness of the solutions and their continuity with respect to the data. The general input forces due to the patches allow well-posedness for the weak form of the equations in the case of strong damping.

\section{Approximation Scheme}

The weak form of the equations of motion can be found by integration by parts of the strong form. For a complete derivation, see the technical report version of [1]. The weak formulation is:

$$
\left\{\begin{aligned}
\int_{\Omega_{\mathcal{B}}} & \hat{\rho} \ddot{U} \phi_{1}=\int_{\Omega_{\mathcal{B}}} \frac{q_{x}}{b} \phi_{1} \\
& -\int_{\Omega_{\mathcal{B}}} \hat{E}(2-2 \hat{\nu}) U_{, x} \phi_{1, x}-\int_{\Omega_{\mathcal{B}}} \hat{E}(1-2 \hat{\nu})\left(U_{, y}+V_{, x}\right) \phi_{1, y}-2 \int_{\Omega_{\mathcal{B}}} \hat{E} \hat{\nu} V_{, y} \phi_{1, x} \\
& -\int_{\Omega_{\mathcal{B}}} \hat{C}_{D}(2-2 \hat{\nu}) \dot{U}_{, x} \phi_{1, x}-\int_{\Omega_{\mathcal{B}}} \hat{C}_{D}(1-2 \hat{\nu})\left(\dot{U}_{, y}+\dot{V}_{, x}\right) \phi_{1, y}-2 \int_{\Omega_{\mathcal{B}}} \hat{C}_{D} \hat{\nu} \dot{V}_{, y} \phi_{1, x} \\
\int_{\Omega_{\mathcal{B}}} & \hat{\rho} \ddot{V} \phi_{2}=\int_{\Omega_{\mathcal{B}}} \frac{q_{y}}{b} \phi_{2} \\
& -\int_{\Omega_{\mathcal{B}}} \hat{E}(2-2 \hat{\nu}) V_{, y} \phi_{2, y}-\int_{\Omega_{\mathcal{B}}} \hat{E}(1-2 \hat{\nu})\left(U_{, y}+V_{, x}\right) \phi_{2, x}-2 \int_{\Omega_{\mathcal{B}}} \hat{E} \hat{\nu} U_{, x} \phi_{2, y} \\
& -\int_{\Omega_{\mathcal{B}}} \hat{C}_{D}(2-2 \hat{\nu}) \dot{V}_{, y} \phi_{2, y}-\int_{\Omega_{\mathcal{B}}} \hat{C}_{D}(1-2 \hat{\nu})\left(\dot{U}_{, y}+\dot{V}_{, x}\right) \phi_{2, x}-2 \int_{\Omega_{\mathcal{B}}} \hat{C}_{D} \hat{\nu} \dot{U}_{, x} \phi_{2, y}
\end{aligned}\right.
$$

for all $\phi_{1}, \phi_{2}$ in $\mathrm{V}=H_{L}^{1}\left(\Omega_{\mathcal{B}}\right) \times H_{L}^{1}\left(\Omega_{\mathcal{B}}\right)$ where we define $H_{L}^{1}\left(\Omega_{\mathcal{B}}\right)=\left\{\psi \in H^{1}\left(\Omega_{\mathcal{B}}\right) \mid \psi=0\right.$ on $\left.\Gamma\right\}$.

To approximate the solutions $(U, V)$ to Eq. (4), we choose basis functions $\mathcal{B}_{u_{k}}(x, y)$ and $\mathcal{B}_{v_{k}}(x, y)$ in $\mathrm{V}$ and form

$$
\begin{aligned}
U^{N}(t, x, y) & =\sum_{k=1}^{\mathcal{N}_{u}} U_{k}(t) \mathcal{B}_{u_{k}}(x, y) \\
V^{N}(t, x, y) & =\sum_{k=1}^{\mathcal{N}_{v}} V_{k}(t) \mathcal{B}_{v_{k}}(x, y)
\end{aligned}
$$

where $\mathcal{B}_{u_{k}}(x, y)$ and $\mathcal{B}_{v_{k}}(x, y)$ can be of the form

$$
\begin{aligned}
& \mathcal{B}_{u_{k}}(x, y)=g_{u_{k}}(x) h_{u_{k}}(y) \\
& \mathcal{B}_{v_{k}}(x, y)=g_{v_{k}}(x) h_{v_{k}}(y)
\end{aligned}
$$




\subsection{Basis Functions}

Linear splines are used to approximate both horizontal and vertical displacements. We consider a uniform partition along both x-axis and y-axis with $N_{x}$ and $N_{y}$ grid points in the respective directions. We designate the length of the structure $l$ and its height $h$. The grid points are defined by $X_{n}=n_{x} h_{x}$ and $Y_{n}=n_{y} h_{y}$ where $h_{x}=l / N_{x}, h_{y}=h /\left(N_{y}-1\right)$, $n_{x}=1, \cdots, N_{x}$ and $n_{y}=1, \cdots N_{y}$.

The linear splines are

$$
g_{n_{x}}=\frac{1}{h_{x}}\left\{\begin{array}{cl}
\left(x-x_{n_{x}-1}\right), & x \in\left[x_{n_{x}-1}, x_{n_{x}}\right] \\
\left(x_{n_{x}+1}-x\right), & x \in\left[x_{n_{x}}, x_{n_{x}+1}\right] \\
0, & \text { otherwise }
\end{array}\right.
$$

and

$$
h_{n_{y}}=\frac{1}{h_{y}}\left\{\begin{array}{cl}
\left(y-y_{n_{y}-1}\right), & y \in\left[y_{n_{y}-1}, y_{n_{y}}\right] \\
\left(y_{n_{y}+1}-y\right), & y \in\left[y_{n_{y}}, y_{n_{y}+1}\right] . \\
0, & \text { otherwise }
\end{array}\right.
$$

In general, the standard splines are modified to satisfy the essential boundary conditions. In the present case, the fixed displacements and slope condition for $(x=0)$ is satisfied by setting the appropriate spline contributions to zero. For the computations, the corresponding grid points are not included in the discretized system. They are, however, included in the definition of $h_{x}$.

\subsection{Finite Dimensional System}

With bases defined in Eq. (5), the approximating subspaces are taken to be $H_{u}^{N}=\operatorname{span}\left\{\mathcal{B}_{u_{k}}\right\}_{k=1}^{\mathcal{N}_{u}}$ and $H_{v}^{N}=\operatorname{span}\left\{\mathcal{B}_{v_{k}}\right\}_{k=1}^{\mathcal{N}_{v}}$. We note that $H^{N}=H_{u}^{N} \times H_{v}^{N} \subset V$. The approximating system is then determined by restricting the weak form in Eq. (4) to $H^{N}$ with basis functions used as test functions. We obtain 


$$
\begin{aligned}
& \iint_{\Omega_{\mathcal{B}}} \sum_{k=1}^{\mathcal{N}_{u}} \hat{E}(2-2 \hat{\nu}) U_{k}(t) \mathcal{B}_{u_{k}, x} \mathcal{B}_{u_{j}, x}+\int_{\Omega_{\mathcal{B}}} \sum_{k=1}^{\mathcal{N}_{u}} \hat{E}(1-2 \hat{\nu}) U_{k}(t) \mathcal{B}_{u_{k}, y} \mathcal{B}_{u_{j}, y} \\
& +\int_{\Omega_{\mathcal{B}}} \sum_{k=1}^{\mathcal{N}_{u}} \hat{E}(1-2 \hat{\nu}) V_{k}(t) \mathcal{B}_{v_{k}, x} \mathcal{B}_{u_{j}, y}+2 \int_{\Omega_{\mathcal{B}}} \sum_{k=1}^{\mathcal{N}_{u}} \hat{E} \hat{\nu} V_{k}(t) \mathcal{B}_{v_{k}, y} \mathcal{B}_{u_{j}, x} \\
& +\int_{\Omega_{\mathcal{B}}} \sum_{k=1}^{\mathcal{N}_{u}} \hat{C}_{D}(2-2 \hat{\nu}) \dot{U}_{k}(t) \mathcal{B}_{u_{k}, x} \mathcal{B}_{u_{j}, x}+\int_{\Omega_{\mathcal{B}}} \sum_{k=1}^{\mathcal{N}_{u}} \hat{C}_{D}(1-2 \hat{\nu}) \dot{U}_{k}(t) \mathcal{B}_{u_{k}, y} \mathcal{B}_{u_{j}, y} \\
& +\int_{\Omega_{\mathcal{B}}} \sum_{k=1}^{\mathcal{N}_{u}} \hat{C}_{D}(1-2 \hat{\nu}) \dot{V}_{k}(t) \mathcal{B}_{v_{k}, x} \mathcal{B}_{u_{j}, y}+2 \int_{\Omega_{\mathcal{B}}} \sum_{k=1}^{\mathcal{N}_{u}} \hat{C}_{D} \hat{\nu} \dot{V}_{k}(t) \mathcal{B}_{v_{k}, y} \mathcal{B}_{u_{j}, x} \\
& +\int_{\Omega_{\mathcal{B}}} \sum_{k=1}^{\mathcal{N}_{u}} \hat{\rho} \ddot{U}_{k}(t) \mathcal{B}_{u_{k}} \mathcal{B}_{u_{j}}=\int_{\Omega_{\mathcal{B}}} \frac{q_{x}}{b} \mathcal{B}_{u_{j}} \\
& \int_{\Omega_{\mathcal{B}}} \sum_{k=1}^{\mathcal{N}_{v}} \hat{E}(2-2 \hat{\nu}) V_{k}(t) \mathcal{B}_{v_{k}, y} \mathcal{B}_{v_{j}, y}+\int_{\Omega_{\mathcal{B}}} \sum_{k=1}^{\mathcal{N}_{v}} \hat{E}(1-2 \hat{\nu}) V_{k}(t) \mathcal{B}_{v_{k}, x} \mathcal{B}_{v_{j}, x} \\
& +\int_{\Omega_{\mathcal{B}}} \sum_{k=1}^{\mathcal{N}_{v}} \hat{E}(1-2 \hat{\nu}) U_{k}(t) \mathcal{B}_{u_{k}, y} \mathcal{B}_{v_{j}, x}+2 \int_{\Omega_{\mathcal{B}}} \sum_{k=1}^{\mathcal{N}_{v}} \hat{E} \hat{\nu} U_{k}(t) \mathcal{B}_{u_{k}, x} \mathcal{B}_{v_{j}, y} \\
& +\int_{\Omega_{\mathcal{B}}} \sum_{k=1}^{\mathcal{N}_{v}} \hat{C}_{D}(2-2 \hat{\nu}) \dot{V}_{k}(t) \mathcal{B}_{v_{k}, y} \mathcal{B}_{v_{j}, y}+\int_{\Omega_{\mathcal{B}}} \sum_{k=1}^{\mathcal{N}_{v}} \hat{C}_{D}(1-2 \hat{\nu}) \dot{V}_{k}(t) \mathcal{B}_{v_{k}, x} \mathcal{B}_{v_{j}, x} \\
& +\int_{\Omega_{\mathcal{B}}} \sum_{k=1}^{\mathcal{N}_{v}} \hat{C}_{D}(1-2 \hat{\nu}) \dot{U}_{k}(t) \mathcal{B}_{u_{k}, y} \mathcal{B}_{v_{j}, x}+2 \int_{\Omega_{\mathcal{B}}} \sum_{k=1}^{\mathcal{N}_{v}} \hat{C}_{D} \hat{\nu} \dot{U}_{k}(t) \mathcal{B}_{u_{k}, x} \mathcal{B}_{v_{j}, y} \\
& +\int_{\Omega_{\mathcal{B}}} \sum_{k=1}^{\mathcal{N}_{v}} \hat{\rho} \ddot{V}_{k}(t) \mathcal{B}_{v_{k}} \mathcal{B}_{v_{j}}=\int_{\Omega_{\mathcal{B}}} \frac{q_{y}}{b} \mathcal{B}_{v_{j}}
\end{aligned}
$$

\subsection{Matrix System}

To form the matrix system corresponding to Eq. (9), we define the displacement vectors

$$
\mathcal{U}^{\mathcal{N}_{u}}(t)=\left[\begin{array}{c}
u_{1}(t) \\
\vdots \\
u_{\mathcal{N}_{u}}(t)
\end{array}\right], \mathcal{V}^{\mathcal{N}_{v}}(t)=\left[\begin{array}{c}
v_{1}(t) \\
\vdots \\
v_{\mathcal{N}_{v}}(t)
\end{array}\right]
$$

The generalized displacements are $\mathcal{W}^{\mathcal{N}}(t)=\left[\mathcal{U}^{\mathcal{N}}(t), \mathcal{V}^{\mathcal{N}_{v}}(t)\right]^{T}$, where $\mathcal{N}=\mathcal{N}_{u}+\mathcal{N}_{v}$. 
We define the submatrices

$$
\begin{array}{ll}
{\left[U_{M}\right]_{k, j}=\int_{\Omega_{\mathcal{B}}} \hat{\rho} \mathcal{B}_{u_{k}} \mathcal{B}_{u_{j}}} & {\left[V_{M}\right]_{k, j}=\int_{\Omega_{\mathcal{B}}} \hat{\rho} \mathcal{B}_{v_{k}} \mathcal{B}_{v_{j}}} \\
{\left[U_{11}\right]_{k, j}=\int_{\Omega_{\mathcal{B}}} \hat{E}(2-2 \hat{\nu}) \mathcal{B}_{u_{k}, x} \mathcal{B}_{u_{j}, x}} & {\left[V_{21}\right]_{k, j}=\int_{\Omega_{\mathcal{B}}} \hat{E}(2-2 \hat{\nu}) \mathcal{B}_{v_{k}, y} \mathcal{B}_{v_{j}, y}} \\
{\left[V_{11}\right]_{k, j}=\int_{\Omega_{\mathcal{B}}} 2 \hat{E} \hat{\nu} \mathcal{B}_{v_{k}, y} \mathcal{B}_{u_{j}, x}} & {\left[U_{21}\right]_{k, j}=\int_{\Omega_{\mathcal{B}}} 2 \hat{E} \hat{\nu} \mathcal{B}_{u_{k}, x} \mathcal{B}_{v_{j}, y}} \\
{\left[U_{12}\right]_{k, j}=\int_{\Omega_{\mathcal{B}}} \hat{E}(1-2 \hat{\nu}) \mathcal{B}_{u_{k}, y} \mathcal{B}_{u_{j}, y}} & {\left[V_{22}\right]_{k, j}=\int_{\Omega_{\mathcal{B}}} \hat{E}(1-2 \hat{\nu}) \mathcal{B}_{v_{k}, x} \mathcal{B}_{v_{j}, x}} \\
{\left[V_{12}\right]_{k, j}=\int_{\Omega_{\mathcal{B}}} \hat{E}(1-2 \hat{\nu}) \mathcal{B}_{v_{k}, x} \mathcal{B}_{u_{j}, y}} & {\left[U_{22}\right]_{k, j}=\int_{\Omega_{\mathcal{B}}} \hat{E}(1-2 \hat{\nu}) \mathcal{B}_{u_{k}, y} \mathcal{B}_{v_{j}, x}} \\
{\left[F_{u}\right]_{j}=\int_{\Omega_{\mathcal{B}}} \frac{q_{x}}{b} \mathcal{B}_{u_{j}}} & {\left[F_{v}\right]_{j}=\int_{\Omega_{\mathcal{B}}} \frac{q_{y}}{b} \mathcal{B}_{v_{j}}}
\end{array}
$$

Similar expressions define $\left[\tilde{U}_{r s}\right]$ and $\left[\tilde{V}_{r s}\right]$ for $r, s=1,2$ with $\hat{C}_{D}$ replacing $\hat{E}$.

With the definitions in Eq. (11), the complete matrix system can be formed. The mass, stiffness and damping matrices and the forcing vector have the form

$$
\begin{gathered}
M^{\mathcal{N}}=\left[\begin{array}{c|c}
U_{M} & 0 \\
\hline 0 & V_{M}
\end{array}\right], \\
K_{E}^{\mathcal{N}}=\left[\begin{array}{c|c}
U_{11}+U_{12} & V_{11}+V_{12} \\
\hline U_{21}+U_{22} & V_{21}+V_{22}
\end{array}\right], \\
K_{C_{D}}^{\mathcal{N}}=\left[\begin{array}{c|c}
\tilde{U}_{11}+\tilde{U}_{12} & \tilde{V}_{11}+\tilde{V}_{12} \\
\hline \tilde{U}_{21}+\tilde{U}_{22} & \tilde{V}_{21}+\tilde{V}_{22}
\end{array}\right]
\end{gathered}
$$

and

$$
F^{\mathcal{N}}(t)=\left[\begin{array}{c}
F_{u}(t) \\
F_{v}(t)
\end{array}\right]
$$

Writing the system in the first order form, we obtain

$$
\left[\begin{array}{cc}
K_{E}^{\mathcal{N}} & 0 \\
0 & M^{\mathcal{N}}
\end{array}\right]\left[\begin{array}{l}
\dot{\mathcal{W}}(t) \\
\ddot{\mathcal{W}}(t)
\end{array}\right]=\left[\begin{array}{cc}
0 & K_{E}^{\mathcal{N}} \\
-K_{E}^{\mathcal{N}} & -K_{C_{D}}^{\mathcal{N}}
\end{array}\right]\left[\begin{array}{l}
\mathcal{W}(t) \\
\dot{\mathcal{W}}(t)
\end{array}\right]+\left[\begin{array}{c}
0 \\
F^{\mathcal{N}}(t)
\end{array}\right]
$$

In order to numerically solve the system in Eq. (14), it is necessary to evaluate the submatrices in Eq. (11). For simplicity, the number of elements is chosen identical for $U^{N}$ and $V^{N}$ in Eq. (5); that is $\mathcal{N}_{u}=\mathcal{N}_{v}$. 


\subsection{Approximation of Natural Frequencies and Modes}

Both mathematical formulations for the equations of motion in Eq. (1) and (4) include KelvinVoigt damping. It is well known [23] that, for a periodic excitation with a frequency at or near a natural frequency, damping is of primary importance and must be taken into account. However, the influence of a small amount of damping upon the response of a system during an excitation of short duration is not likely to be significant.

The damage detection method we propose in this report uses the fact that a small local damage to a structure will induce changes in the natural frequencies even if the global physical properties remain unchanged. Only the frequency shifts due to damage obtained from the vibration response after an excitation of short duration are actually used for the characterization, not the values of the natural frequencies themselves. We tacitly assume that, in the event of a small localized damage, the natural frequency shifts are identical whether strong damping is included in the physical model or not. The main advantage of an identification method that does not take into account damping is its computational simplicity. For instance, the approximate natural frequencies and modes of the structures depicted in Fig. 1 can be obtained by considering the undamped, unforced approximate system in Eq. (14)

$$
M^{\mathcal{N}} \ddot{\mathcal{W}}(t)+K_{E}^{\mathcal{N}} \mathcal{W}(t)=0
$$

which yields the generalized eigenvalue problem

$$
K_{E}^{\mathcal{N}} \mathcal{W}(t)=\omega^{2} M^{\mathcal{N}} \mathcal{W}(t)
$$

The system in Eq. (16) has $\mathcal{N}$ eigenvalues $\omega_{i}$. The natural frequencies are obtained by

$$
f_{i}=\omega_{i} /(2 \pi) .
$$

In the matlab computing environment that we used, the eigenvalue problem can be readily solved.

\section{Experimental Results}

\subsection{Specimens, Procedure and Experimental Setup}

\subsubsection{Specimens: Description and Preparation}

A series of experiments were conducted to determine the time resolved vibration response of cantilever structures described in Fig. 1. As depicted in Fig. 2, piezoelectric ceramic patches were symmetrically bonded to each side of aluminum plates. This arrangement, shown in Fig. 3, allowed us to excite the specimens in a pure bending mode by actuating the patches out-of-phase. Longitudinal modes can also be excited by actuating both patches in-phase. However, in the experiments described in this report, only the pure bending modes were used. The specimens consisted of $304.8 \times 25.4 \times 1.016(\mathrm{~mm}) 2024-\mathrm{T} 3$ aluminum slabs. The patches were $25.4 \times 25.4 \times 0.0508(\mathrm{~mm})$ lead zirconate titanates, appropriate for large displacement applications. Both aluminum and ceramic surfaces were lightly sanded to roughen their surfaces and cleaned with trichloroethane. They were bonded with a low viscosity, room temperature cure epoxy adhesive. Electrical leads were soldered on the outside surface of the patches. 
Aluminum 2024-T3

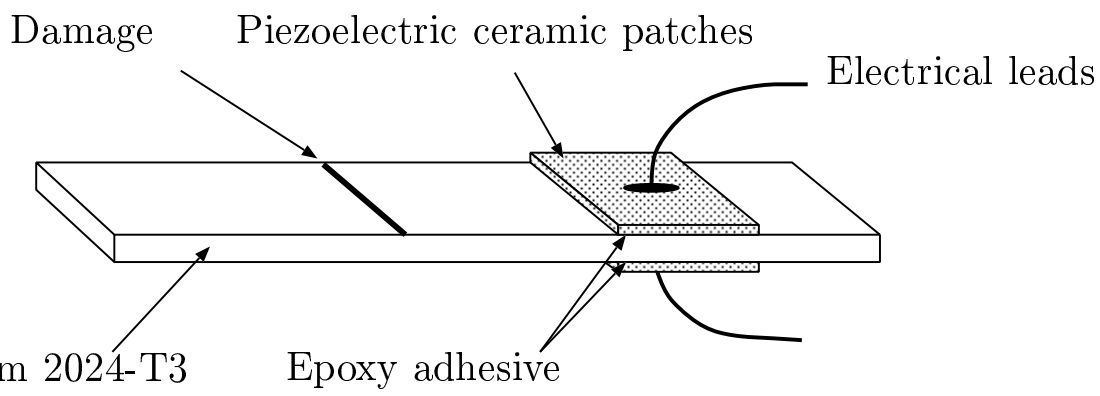

Figure 2: Beam with bonded piezoelectric ceramic patches

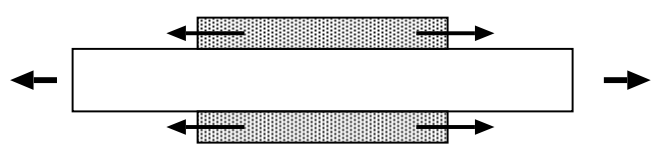

In-phase longitudinal mode

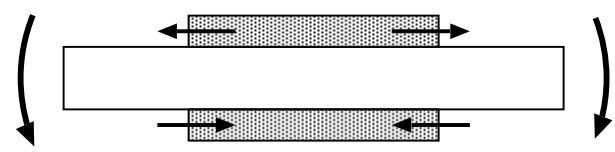

Out-of-phase transverse mode

Figure 3: In- and out-of-phase excitation modes

\subsubsection{Experimental Setup}

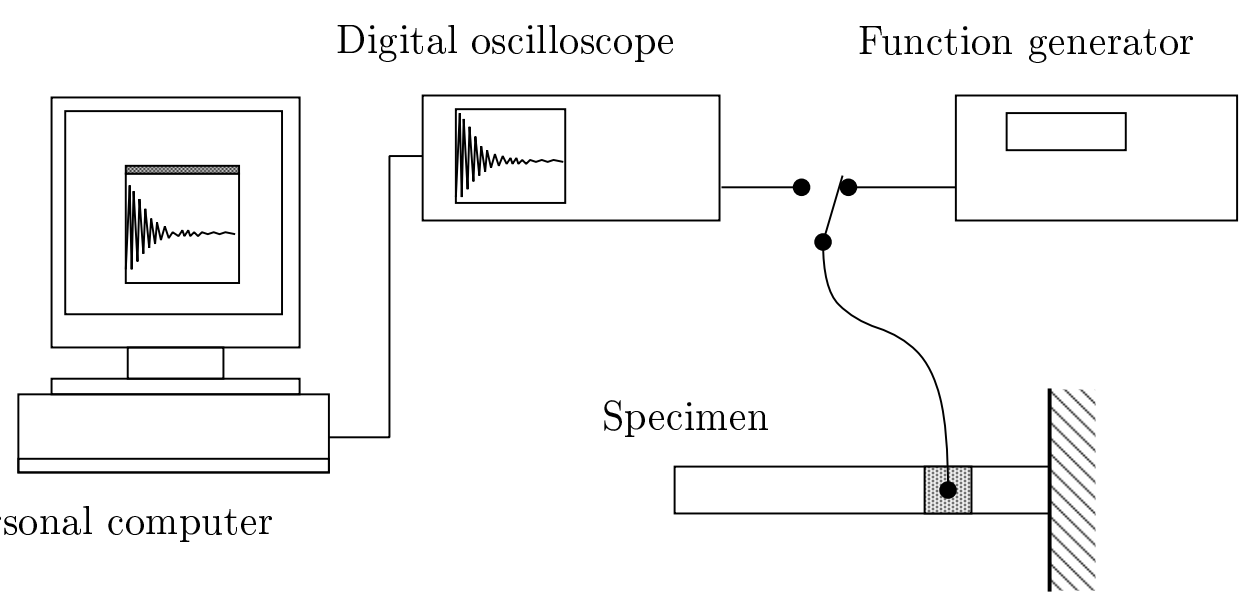

Figure 4: Experimental setup

Fig. 4 depicts a diagram of the experimental setup used to determine the time resolved vibration response of plate-like structures. The specimens were clamped in a vise. A function generator provided the excitation to the patches. The piezoelectric patches were excited with a $2 \mathrm{~ms}$ square pulse signal. An oscilloscope was used to digitize the electrical signal generated by the deformation of the structure. A set of diodes, acting as a switch, was used to allow the input signal to the patches and their electrical response to the oscilloscope without interference. A personal computer allowed the storage of the data for further processing. 


\subsubsection{Production of Damage}

As depicted in Fig. 2, the damage was produced by machining a rectangular groove at different locations. Table 1 summarizes the damage locations and depths for every damaged specimen. The measurements of locations were made from the cantilever end. Specimens 14, 8, 7 and 9 featured an identical damage located at increasing distance from the cantilever end. Specimens 13, 11 and 14 featured damage of increasing severity at the same location. The two sets were designed to test the ability of the identification method to estimate correctly a damage location and severity.

\begin{tabular}{|c||c|c|c|}
\hline Specimen & Location of defect $(\mathrm{mm})$ & Depth $(\mathrm{mm})$ & Width $(\mathrm{mm})$ \\
\hline \hline 7 & 176.3 & 0.42 & 1.64 \\
\hline 8 & 138.7 & 0.44 & 1.65 \\
\hline 9 & 214.4 & 0.42 & 1.64 \\
\hline 11 & 101.3 & 0.33 & 1.63 \\
\hline 13 & 101.1 & 0.25 & 1.64 \\
\hline 14 & 101.6 & 0.43 & 1.64 \\
\hline
\end{tabular}

Table 1: Location and depth of damage

\subsubsection{Experimental Procedures and Measurement Method}

For each undamaged specimen, a baseline measurement of the dynamic response to a $2 \mathrm{~ms}$ square pulse input was collected five consecutive times. The specimens were then damaged and the measurements repeated again five times. To illustrate the flexibility of the detection method, the specimens were typically unclamped and reclamped between each data acquisition. Once stored in computer memory, each time resolved vibration response was processed by using a Fast Fourier Transform algorithm to obtain a frequency spectrum. The natural frequencies were determined from the peak locations. For every specimen, two sets (undamaged structure responses and damaged structure responses) of five measurements of the natural frequencies were obtained. Fig. 5 shows, as an example, the frequency spectra for specimen 14. The graph also displays in detail the sixth harmonic before and after damage was applied to the specimen.

For every specimen, the five measured values corresponding to the same harmonic were averaged for both undamaged and damaged cases. The standard deviations of the measurements were also computed to characterize their distribution.

\subsection{Discussions}

\subsubsection{Validation of Smart Actuation/Sensing Capabilities}

To validate the results obtained in the smart actuation/sensing mode, the results obtained by using the patches in self actuator/sensor mode were compared to results from experiments 


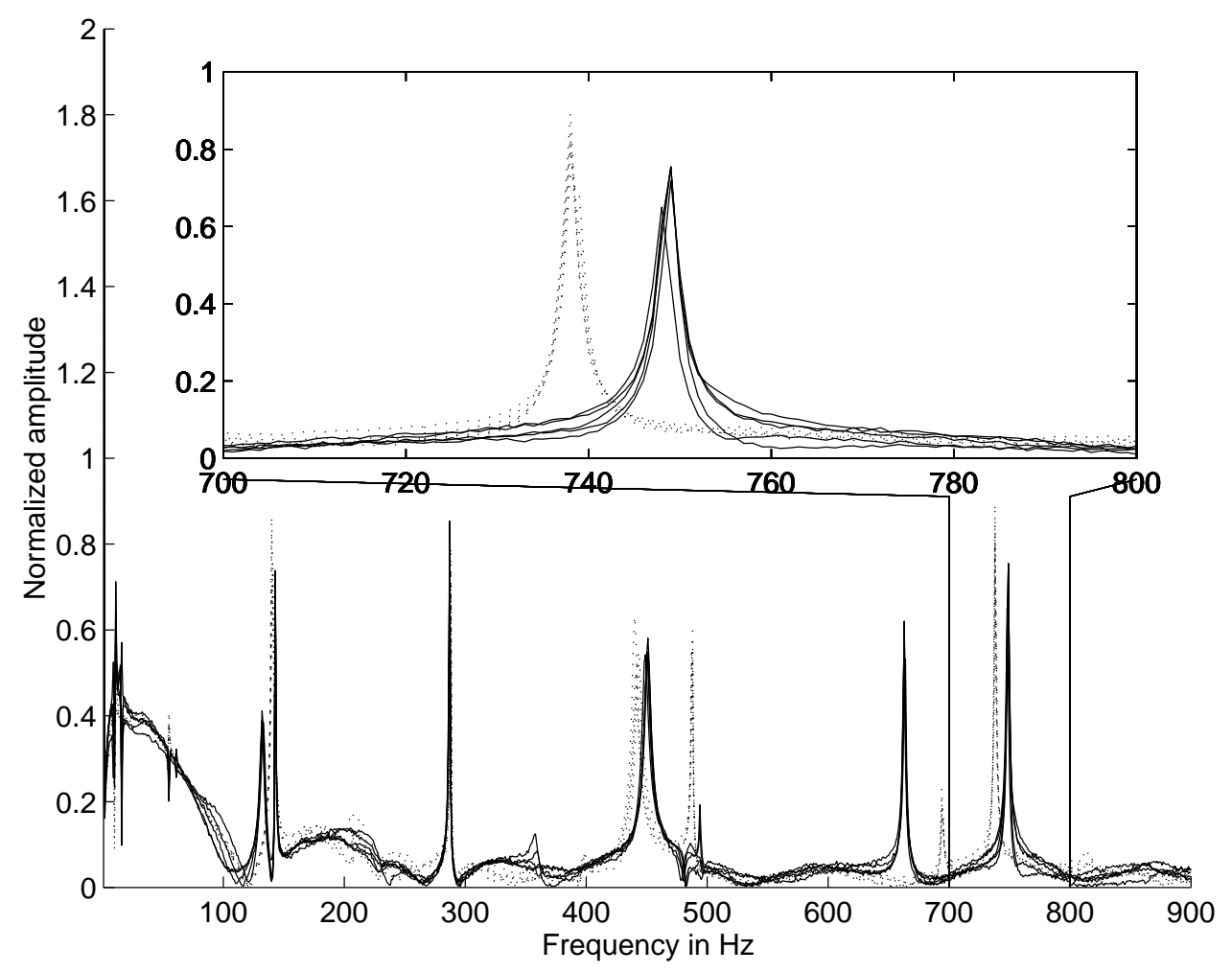

Figure 5: Natural frequency peaks for specimen 14 before (solid lines) and after damage (dotted lines).

conducted in the passive sensing mode with excitations provided by hammer hits. An undamaged specimen was excited by hammer hits and allowed to vibrate freely. The data was collected after each hit. Vibration data was also collected when the excitation was provided by the piezoelectric patches. Fig. 6 shows the frequency spectrum of typical data sets for piezo-actuation and hammer hit. The peaks indicate the natural frequencies of the structure. The data for both types of excitation, external and self actuating/sensing, is similar in spite of relative peak intensity differences.

\subsubsection{Measurements on Undamaged Specimens, Influence of Clamping Strength}

To evaluate the influence of changes in clamping strength and position, five dynamic tests were conducted with specimen 8 left clamped at all times. The same tests were repeated with specimen 8 released and reclamped between each data acquisition. The results are displayed in Table 2. The standard deviations of the natural frequency measurements for the reclamped case are larger. This is expected since natural frequencies depend on the total length of the specimens. Every reclamping introduces an uncertainty which is reflected by the increase of the standard deviation. However, the average values for both experiments are in very good agreement. These results suggest that the clamping device used for the experiments allows reliable measurements. This is particularly important since the specimens had to be unclamped to machine the damage and reclamped in position for dynamic testing.

This experiment also illustrates the flexibility of self-analyzing frequency measurement meth- 


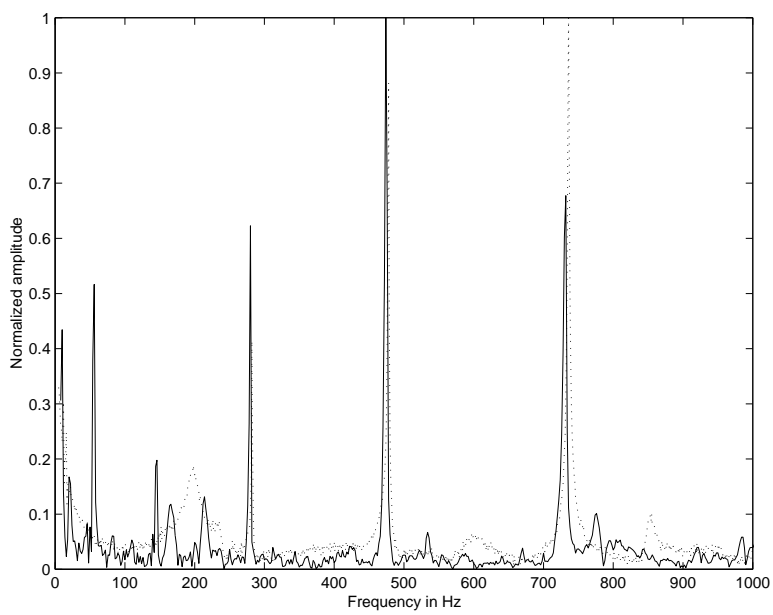

Figure 6: Normalized frequency spectra of dynamic responses of specimen 5 to piezo-excitation (solid lines) and hammer hit (dotted lines)

ods with piezo-electric patches, which we find suitable for industrial applications where parts can be frequently disassembled.

\begin{tabular}{|c||c|c|}
\hline Harmonic & Frequency in $\mathrm{Hz}$ (clamped) & Frequency in $\mathrm{Hz}$ (reclamped) \\
\hline \hline 1 & $11.0 \pm 0.0$ & $10.6 \pm 0.5$ \\
\hline 2 & $55.0 \pm 0.0$ & $55.0 \pm 0.0$ \\
\hline 3 & $143.0 \pm 0.0$ & $142.6 \pm 0.5$ \\
\hline 4 & $285.0 \pm 0.0$ & $284.6 \pm 0.5$ \\
\hline 5 & $490.4 \pm 0.5$ & $489.6 \pm 0.5$ \\
\hline 6 & $746.0 \pm 0.0$ & $745.2 \pm 1.1$ \\
\hline 7 & $1033.0 \pm 0.0$ & $1031.4 \pm 1.3$ \\
\hline
\end{tabular}

Table 2: Average values and standard deviations of natural frequency measurements for undamaged specimen 8

\section{Identification Results}

The identification problem is formulated as the problem of determining estimates of parameters such as the location and size of the damage given data measurements $z$. In this work, we assume that a finite dimensional $\mathcal{N}$ approximation based on a grid with points $\left(N_{x}, N_{y}\right)$ as described in Section 2 has been made. We then assume that the defect is represented by removing $N_{\text {depth }}$ elements in a single column located between the grid point $N_{\text {pos }}$ and $N_{\text {pos }}+1$. The width of the simulated defect is therefore given by the size of the elements. Fig. 7 depicts an example of the finite element grid in the damaged case with $N_{\text {depth }}=2$.

In the experimental results reported here, the data consisted of the natural frequency shifts 
obtained from the dynamic tests described in Section 3.

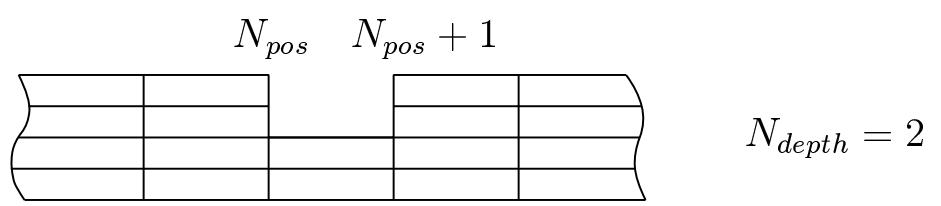

Figure 7: Finite Element Grid around a Damage for a Plate-Like Structure

We let $q=\left(N_{\text {pos }}, N_{\text {depth }}\right)$ and assume that $q \in Q$ where $Q$ is an admissible parameter space. We seek $\bar{q} \in Q$ which minimizes

$$
J^{\mathcal{N}}(q)=\sum_{j=1}^{N_{\text {har }}} C_{j}\left|f_{j}^{\mathcal{N}}(q)-z_{j}\right|^{2}
$$

where $N_{\text {har }}$ is the number of harmonics used to solve the identification problem. In the experiments presented in this report, the first seven harmonics where measured $\left(N_{\text {har }}=7\right)$. The value $f_{j}^{\mathcal{N}}(q)$ of the natural frequency shifts for the $j^{\text {th }}$ harmonic is given by solving the generalized eigenvalue problem in Eq. (16) with given discretization $\mathcal{N}$. Here, $z_{j}$ is the natural frequency shift observed for the $j^{\text {th }}$ harmonic. A weight coefficient $C_{j}$ is associated with each harmonic. A cost function using only higher order natural frequency shifts is not adequate to obtain a unique solution to the damage detection problem. The non-uniqueness of the solutions to the damage location problem is a difficulty also encountered in methods based on 1-D models of the structures [14]. Higher natural frequencies can provide better accuracy since they are sensitive to local phenomena. However, they can lead to multiple solutions. That is, there exists more than one set of damage parameters $q$ which leads to the same value of the cost function. Lower order frequencies are sensitive to global changes in a structure and do not lead to multiple solutions. However, because the frequency shifts due to damage are smaller for lower natural frequencies, a precise fit is difficult and the estimated positions are not accurate.

To alleviate these difficulties, we propose a two step damage detection method:

- A first optimization with a cost function based on lower order frequency shifts to obtain a first estimate of the damage location. That is, we use the cost function (18) with

$$
C_{j}= \begin{cases}1 & j=1,2,3 \\ 0 & j=4,5,6,7\end{cases}
$$

- A second optimization taking into account all the frequency shifts experimentally available $\left(C_{j}=1\right.$ with $\left.j=1, \cdots, 7\right)$, to improve the accuracy of the location within the constraints of the first search. 
To illustrate the method, Fig. 8 allows one to compare both cost functions computed for specimen 14. For these computations, the defects were assumed to have a rectangular shape $1.52 \mathrm{~mm}$ wide and $0.51 \mathrm{~mm}$ deep. The material properties for aluminum used in the simulations were $E=7.310^{10} \mathrm{~Pa}, \rho=2770 \mathrm{~kg} / \mathrm{m}^{3}$ and $\nu=0.3$. The cost function depending on the first three natural frequency shifts indicates a minimum in the vicinity of the actual damage location. The cost function depending on all the frequency shifts experimentally available indicates the damage position with a better accuracy but also displays a local minimum at another location.

In the identification results that follow, minimization of the cost functional (18) was accomplished via a Nelder-Mead optimizer.

Cost function with harmonics 1 to 3

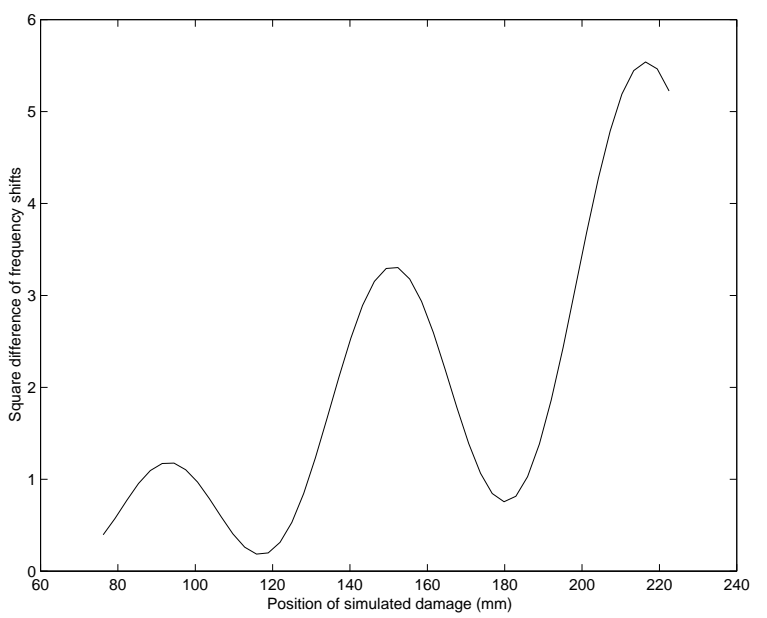

Cost function with harmonics 1 to 7

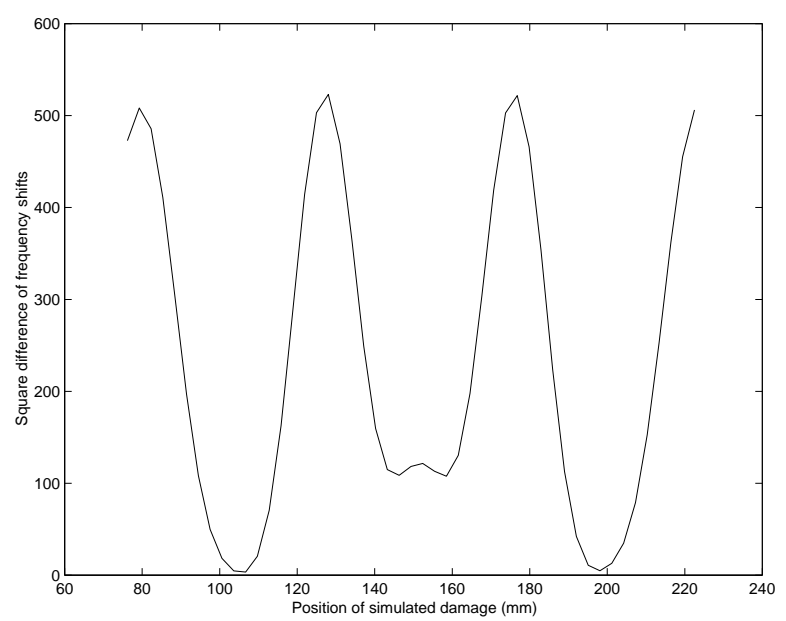

Figure 8: Cost functions for specimen 14 for estimated damage positions between 75 and $225 \mathrm{~mm}$ (actual damage located at $101.6 \mathrm{~mm}$ )

\subsection{Location of Damage}

A series of experiments were conducted with specimens 14, 8, 7 and 9 to test the ability of the method to estimate correctly a damage location. Fig. 9 allows one to compare experimental results with simulated data using the best estimate of damage location from the identification problem for each specimen. For the computations, the finite element grid was chosen (based on test calculations) with $\left(N_{x}, N_{y}\right)=(200,3)$. The corresponding simulated defects had a rectangular shape $1.52 \mathrm{~mm}$ wide and $0.51 \mathrm{~mm}$ deep. The real defect sizes are summarized in Table 1. Each graph in Fig. 9 depicts the natural frequency shifts due to damage for each harmonic. The first seven harmonics are presented and numbered from 1 to 7 . The experimental data is plotted in solid lines with error bars representing the standard deviations. The dotted lines represent the simulated data that optimizes the cost function. Although only the numerical value of a frequency shift for a given harmonic is meaningful, the data is displayed as a continuous line. This allows one to easily compare the data obtained from different specimens. The profiles indicate that higher order natural frequencies have very 
different shift patterns depending on the damage location. Both actual and estimated locations are indicated at the top of each graph. An excellent agreement is obtained for all locations. Fig. 10 depicts graphically the estimated and actual locations of damage for specimens 14, 8, 7 and 9. The vertical dimensions are exaggerated for viewing purposes. The horizontal dimensions maintain the specimens proportions. The actual defect locations are marked inside the structures. The estimated positions are indicated by the arrows. The errors in location are summarized in Table 3. For all specimens, the detection method estimates correctly the damage location.
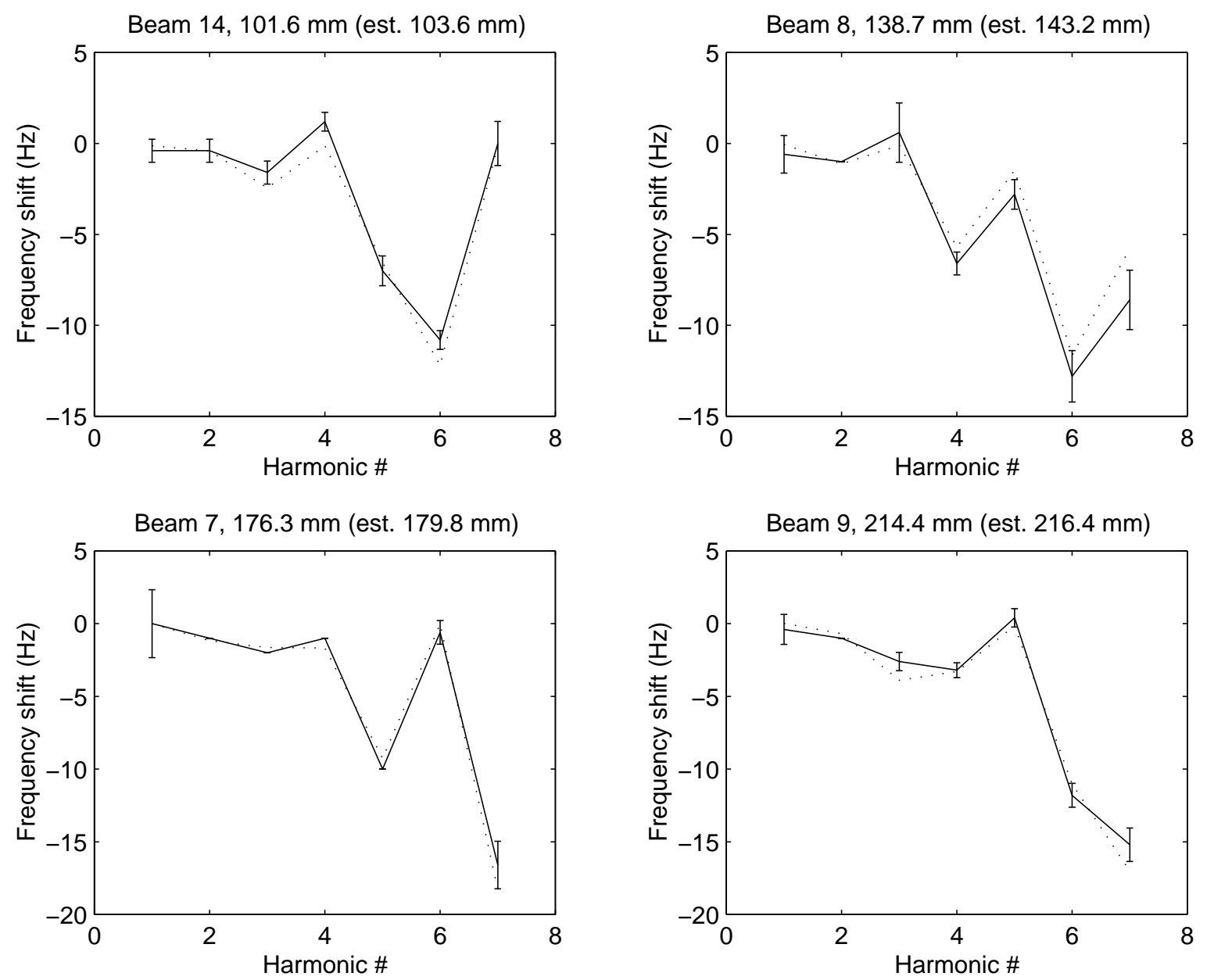

Figure 9: Comparison of experimentally measured (with error bars) and estimated natural frequency shifts (dotted lines)

\subsection{Characterization of Damage}

A series of dynamic tests were conducted on specimens 13, 11 and 14 to characterize our ability to determine the severity of the damage. The specimens had damage of increasing 


\begin{tabular}{|c||c|c|c|}
\hline Specimen & Actual location $(\mathrm{mm})$ & Estimated location $(\mathrm{mm})$ & Error $(\%)$ \\
\hline \hline 14 & 101.6 & 103.6 & 1.97 \\
\hline 8 & 138.7 & 143.2 & 3.24 \\
\hline 7 & 176.3 & 179.8 & 1.98 \\
\hline 9 & 214.4 & 216.4 & 0.93 \\
\hline
\end{tabular}

Table 3: Estimation errors for the location of damage

\begin{tabular}{|c|c|}
\hline 孀: & $103.6 \mathrm{~mm}$ (est.) \\
\hline Specimen 14 & $101.6 \mathrm{~mm}$ \\
\hline 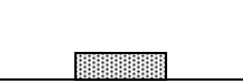 & $143.2 \mathrm{~mm}$ (est.) \\
\hline Specimen 8 & $138.7 \mathrm{~mm}$ \\
\hline 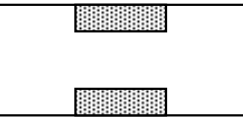 & $\downarrow 179.8 \mathrm{~mm}$ (est.) \\
\hline Specimen 7 & $176.3 \mathrm{~mm}$ \\
\hline 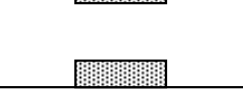 & † $216.4 \mathrm{~mm}$ (est.) \\
\hline Specimen 9 & $214.4 \mathrm{~mm}$ \\
\hline
\end{tabular}

Figure 10: Comparison of actual and estimated location of damage

depths at an identical location. Fig. 11 allows one to compare the experimentally determined natural frequency shifts with the estimated ones for each harmonic. The finite element grid used for these simulations was chosen with $\left(N_{x}, N_{y}\right)=(200,5)$. The corresponding defect had a rectangular shape $1.52 \mathrm{~mm}$ wide with a variable depth depending on the number of elements removed from the undamaged grid. The position of the defect was fixed at $103.6 \mathrm{~mm}$ from the cantilever end, the estimated damage location found for specimen 14. Fig. 11 shows the first seven harmonics. The experimental data is plotted in solid lines (note that the figures have different scales). The dotted lines represent the simulated data.

Table 4 summarizes the results. The extent of the damage is correctly estimated for specimens 11 and 13. For specimen 14, the depth of the damage is overestimated. A decrease in accuracy for the estimation of damage severity when the depth increases is also reported by Hassiotis and Jeong [22]. However, when we consider the extent of damage in terms of cross-sectional area removed, the damage is characterized correctly for all specimens.

\section{Conclusion}

We have presented a nondestructive evaluation technique for damage in beam-like structures based on the experimental determination of both lower and higher modal information. An important feature of the method is the use of piezo-electric ceramic patches to both excite 

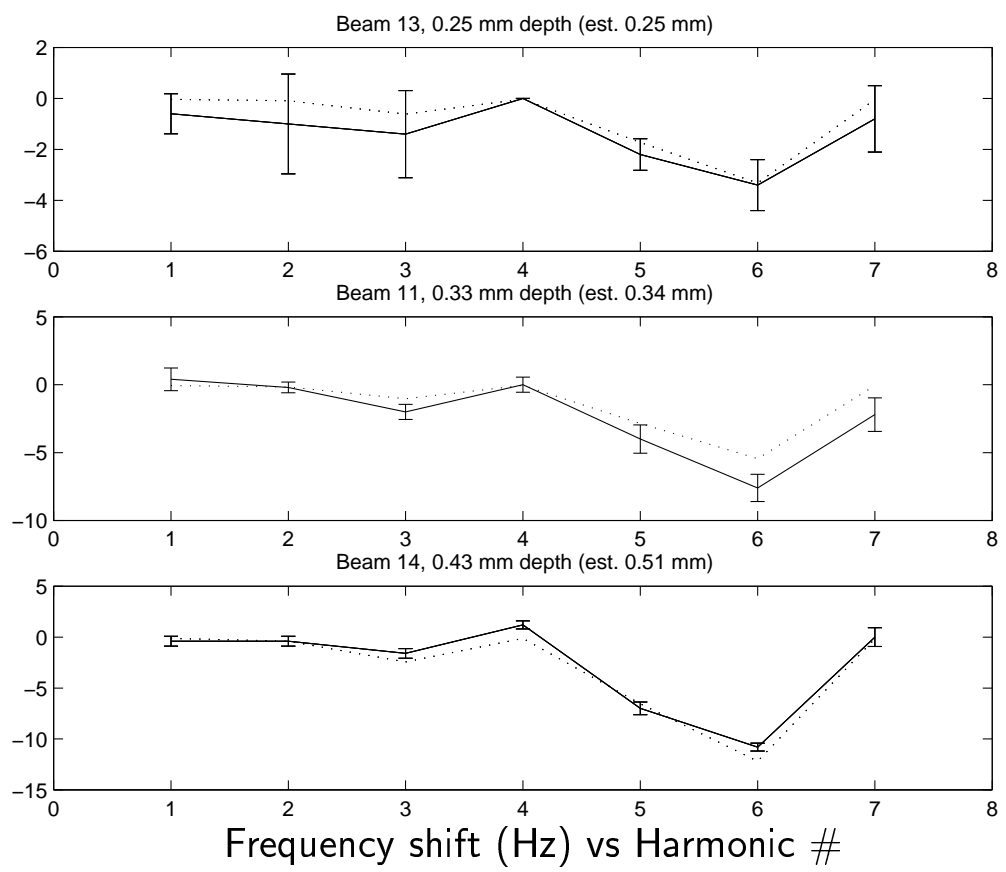

Figure 11: Comparison of experimentally measured (with error bars) and estimated natural frequency shifts (dotted lines)

\begin{tabular}{|c||c|c|c|c|}
\hline Specimen & $\begin{array}{c}\text { Actual depth } \\
(\mathrm{mm})\end{array}$ & $\begin{array}{c}\text { Estimated depth } \\
(\mathrm{mm})\end{array}$ & $\begin{array}{c}\text { Error in depth } \\
(\%)\end{array}$ & $\begin{array}{c}\text { Error in area } \\
(\%)\end{array}$ \\
\hline \hline 13 & 0.25 & 0.25 & 0.0 & 7.3 \\
\hline 11 & 0.33 & 0.34 & 3.0 & 3.7 \\
\hline 14 & 0.43 & 0.51 & 18.6 & 6.9 \\
\hline
\end{tabular}

Table 4: Estimation errors for the severity of damage

and sense vibrations in a self-analysis fashion. Another key feature is the use of a 2-D model to predict the dynamic response of the structures. Most current methods are based on 1-D models, which have known shortcomings.

Using data from dynamic tests performed on aluminum specimens, we demonstrated the feasibility of this approach in successfully detecting and characterizing non-symmetrical damage. The flexibility of the method was further illustrated as the specimens were reclamped between each dynamic test.

Although results were only obtained on aluminum specimens, the framework proposed in this report can be applied to other metallic and composite plate-like or shell structures.

\section{Acknowledgments}

The authors would like to thank Dr. William Winfree, NESB, NASA Langley Research Center, for numerous helpful discussions and for his cooperation in the experimental part of these 
efforts.

\section{References}

[1] H.T. Banks, P. Emeric and L. Plancke, Modeling of Non-Symmetrical Damage in PlateLike Structures, CRSC-TR97-12, NCSU, 1997; Mathematical and Computer Modelling, Vol. 26 n. 3, 1997, pp. 55-65.

[2] L. Keyu, NDT Solution: Interferometric Smart Material for Measuring Permanent Deformations, Materials Evaluation, vol. 54 n. 5, 1996, p. 561.

[3] J. Zelenka, Piezoelectric Resonators and their Applications, Elsevier, 1986.

[4] Nellya N. Rogacheva, The Theory of Piezoelectric Shells and Plates, CRC Press, 1994.

[5] H.T Banks, R.C. Smith and Y. Wang, Smart Material Structures: Modeling, Estimation and Control, Masson/J. Wiley, Paris, Chischester, 1996.

[6] M. Biswas, A.K. Pandey and M.M. Samman, Diagnostic Experimental Spectral/Modal Analysis of a Highway Bridge, The International Journal of Analytical and Experimental Modal Analysis, Vol. 5 n. 1, 1990, pp. 33-42.

[7] S. Alampalli, G. Fu and I. Abdul Aziz, Modal Analysis as a Bridge Inspection Tool, Proceedings, 10th International Modal Analysis Conference, San Diego, CA, Vol. 2, 1992, pp. 1359-1366.

[8] R.G. Flesch, W.J. Gerasch and K. Kernbichler, The Significance of System Identification for Diagnostic Dynamic Testing of Bridges, Structural Dynamics, Proceedings of the European Conference on Structural Dynamics - Eurodyn 90, Bochum, Germany, A.A. Balkema, Rotterdam/Brookflied, Vol. 1, 1991, pp. 419-426.

[9] R.L. Liang, J. Hu and F. Choy, Theoretical Studies of Crack-Induced Eigenfrequency Changes on Beam Structures, Journal of Engineering Mechanics, Vol. 118 n. 2, 1992, pp. 384-396.

[10] Y. Narkis, Identification of Crack Location in Vibrating Simply Supported Beams, Journal of Sound and Vibration, Vol. 172 n. 4, 1994, pp. 549-558.

[11] M. Krawczuk and W.M. Ostachowicz, Modelling and Vibration of a Cantilever Composite Beam with a Transverse Open Crack, Journal of Sound and Vibration, Vol. 183 n. 1, 1995, pp. 69-89.

[12] A. Morassi, Crack-Induced Changes in Eigenparameters of Beam Structures, Journal of Engineering Mechanics, Vol. 119 n. 9, 1994, pp. 1798-1803.

[13] W.M. Hassan, Crack Detection from the Variation of the Eigenfrequencies of a Beam on Elastic Foundation, Engineering Fracture Mechanics, Vol. 52 n. 3, 1995, pp. 409-421. 
[14] H.T. Banks, D.J. Inman, D.J. Leo and Y. Wang, An Experimentally Validated Detection Theory in Smart Structures, Journal of Sound and Vibration, Vol. 191 n. 5, 1996, pp. 859880 .

[15] F.D. Ju and M.E. Mimovich, Experimental Diagnosis of Fracture Damage in Structures by the Modal Frequency Method, Transactions of the ASME, Vol. 110, 1988, pp. 456-463.

[16] S. Christides and A.D.S. Barr, One-Dimensional Theory of Cracked Bernoulli-Euler Beams, International Journal of Mechanical Sciences, Vol. 26 n. 11/12, 1984, pp. 639-648.

[17] P. Cawley and R. Ray, A Comparison of the Natural Frequency Changes Produced by Cracks and Slots, Transactions of the ASME, Vol. 110, 1988, pp. 366-370.

[18] O.N.L. Abraham and J.A. Brandon, The Modelling of the Opening and Closure of a Crack, Journal of Vibration and Acoustics, Vol. 117, 1995, pp. 370-377.

[19] J.A. Brandon and O.N.L. Abraham, Counter-Intuitive Quasi-Periodic Motion in the Autonomous Vibration of Cracked Timoshenko Beams, Journal of Sound and Vibration, Vol. 185 n.3, 1995, pp. 415-430.

[20] P. Gudmundson, The dynamic Behaviour of Slender Structures with Cross-Sectional Cracks, Journal of the Mechanics and Physics of Solids, Vol. 31 n. 4, 1983, pp. 329-345.

[21] G.L. Qian, S.N. Gu and J.S. Jiang, The Dynamic Behaviour and Crack Detection of a Beam with a Crack, Journal of Sound and Vibration, Vol. 138 n. 2, 1990, pp. 233-243.

[22] S. Hassiotis and G.D. Jeong, Assessment of Structural Damage from Natural Frequency Measurements, Computers and Structures, Vol. 49 n. 4, 1993, pp. 679-691.

[23] S. Timoshenko, D.H. Young and W. Weaver, Jr., Vibration Problems in Engineering, John Wiley \& sons, 1974. 